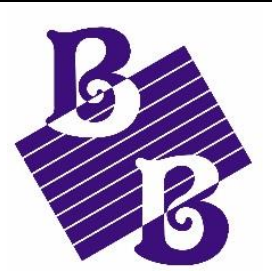

Biobacta
Journal of Bioscience and Applied Research WWW.JBAAR.ORG

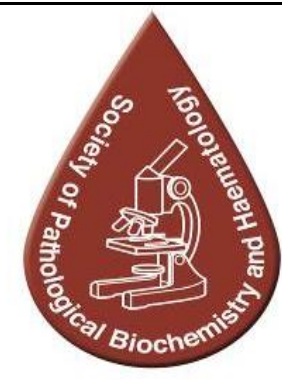

\title{
The Validity of Haematuria and Proteinuria by chemical reagent strip for diagnosis of Schistosoma haematobium infection
}

\author{
Ahmed Abdelhalim Yameny \\ Society of Pathological Biochemistry and Hematology, Egypt \\ Ahmed A. Yameny (Email: dr.ahmedyameny@yahoo.com)
}

DOI: 10.21608/jbaar.2017.126469

\begin{abstract}
Background: Urinary schistosomiasis is a chronic water-borne disease that affects the lives of millions of people globally especially the rural areas of developing countries, Microscopic examination can quantify the intensity of the S.haematobium infection, however, it is relatively insensitive especially in situations involving low-level infections. Hematuria and proteinuria have been considered as alternatives for microscopic testing of urine to diagnose S.haematobium infection, and as a single tool in large-scale control programs. Subjects and methods: This study is a cross-sectional study designed to evaluate haematuria and proteinuria by chemical reagent strip to determine urinary schistosomiasis in the study population, to overall sample was 1000 patients of different ages also, urine microscopic examination was done for detection of S.haematobium eggs by sedimentation centrifugation and nuclepore filtration techniques. Results: The results showed that haematuria by chemical reagent strips had a sensitivity of $27.8 \%$ and specificity of $63.4 \%$. When proteinuria was considered, sensitivity increased to $67.1 \%$ also specificity increased to $80.7 \%$. When both haematuria and proteinuria were considered, sensitivity was $45.2 \%$ and specificity $85.9 \%$. sensitivity in between the previous results. PPVs ranged between $6.13 \%$ and $22.9 \%$ whereas NPVs ranged between $91.1 \%$ and $96.6 \%$. As for diagnostic efficiency, it was best for both haematuria and proteinuria $(83.1 \%)$ followed by proteinuria $79.6 \%$ and least for haematuria (60.7\%), where the area under the curve (AUC) was sufficient for both haematuria and proteinuria 0.61 and good for proteinuria (0.71), where it was not useful for haematuria (0.48). Conclusion and Recommendations: Most previous studies showed blood in urine or microhaematuria is more important than protein and used chemical reagent strips to detect microhaematuria and not protein. While this study showed detect of protein by chemical reagent strip is more important than microhaematuria by chemical reagent strip, and statistical parameters tested were higher with proteinuria than haematuria. The sensitivity, specificity, positive and negative predictive value, the likelihood ratio for positive results, diagnostic efficiency, and area under the curve all were higher in proteinuria than haematuria. The sum of proteinuria and haematuria had more specificity. In this study, we demonstrate that the use of urine reagent strips for both proteinuria and haematuria might be considered for the diagnosis of S. haematobium where microscopy is unavailable.
\end{abstract}

Keywords: Schistosoma haematobium, a chemical reagent strip, proteinuria, haematuria, validity, urinary schistosomiasis. 


\section{Introduction}

Urinary schistosomiasis is a chronic waterborne disease that affects the lives of millions of people globally especially the rural areas of developing countries ${ }^{(1)}$ (Houmsou et al., 2012). Urinary schistosomiasis was reported in Upper Egypt with a prevalence of infection ranging from $4.8 \%$ in Qena to $13.7 \%$ in Fayoum (Abdel-Wahab et al., 2000; El-Khoby et al., 2000). In 2016, due to different control measures, the overall prevalence fell to less than $0.2 \%$, but in 300 villages the prevalence still more than 3\%, so Egypt has started a campaign to reach the final elimination of schistosomiasis by $2020^{(4)}$. Schistosomiasis has been eliminated in Iran, Lebanon, Morocco, and Tunisia with an absence of newly recorded cases in the past few years $(\mathrm{WHO}, 2007)^{(5)}$. The control program in Egypt for Schistosomiasis elimination should be done upon to health education on risk factors with snail control and chemotherapy for infected patients ${ }^{(6)}$.

Microscopic examination can quantify the intensity of the S.haematobium infection, however, it is relatively insensitive especially in situations involving low-level infections ${ }^{(7)}$. Alternative approaches to the parasitologic diagnosis have been tried with some success. One approach comprises an assessment of morbidity, either by ultrasonographic detection of pathologic changes in S.haematobium (bladder) or by chemical reagent strip detection of blood and protein in urine during S.haematobium infection ${ }^{(8,9)}$. Both methods have shown great promise but the need for expensive equipment and comprehensive expertise present a major drawback in the use of ultrasonography in the community-based diagnosis of human schistosomiasis ${ }^{(10)}$. Hematuria and proteinuria have been considered as alternatives for microscopic testing of urine to diagnose S.haematobium infection, and as a single tool in large-scale control programs ${ }^{(11)}$. The presence of reagent strip-detected hematuria and proteinuria has been reported to have high sensitivity and specificity for detecting S.haematobium when used to screen populations in endemic areas and to evaluate response to praziquantil therapy ${ }^{(12)}$.
Haematuria is an important sign of urinary schistosomiasis in endemic areas. Macro -and micro-haematuria may be detected by visual observation or with the aid of a reagent strip ${ }^{(12)}$. Haematuria (HU), Leukocyturia(LU), and Proteinuria (PU) are widely accepted as indirect markers for urinary schistosomiasis in screening procedures ${ }^{(13)}$. Combined semiquantitative data of reagent strip determinations of proteinuria, haematuria, and leukocyturia, the reagent strip index (RSI), provide a more direct measure of acute pathology within the urinary tract ${ }^{(14)}$. These pathologic urine test strip findings correlate with the intensity of infection; however, they are not specific for S.haematobium ${ }^{(15)}$. Chemical reagent strip detection of blood in urine is anticipated to play a central role in the community diagnosis of urinary schistosomiasis, although it is not useful for the diagnosis of other Schistosome spp. infections (16). However, the presence of blood in urine due to menstruation or genitourinary infections are factors that can confound urine reagent strip results ${ }^{(17)}$. Regarding causes of haematuria (RBCs in urine), it may be found in urinary schistosomiasis, genitourinary bacterial infections, acute glomerulonephritis, calculi in the urinary tract, malignancy of the urinary tract, sickle cell disease, leptospirosis, infective endocarditis, and hemorrhagic conditions ${ }^{(18)}$.

Concerning proteinuria, its degree reflects the severity of the glomerular or tubular urinary disease. It should always be considered to indicate underlying disease until proved otherwise also it helps in assessing prognosis and response to treatment ${ }^{(19)}$. The use of sensitivity and specificity of diagnostic tests does not provide the most practical information. Predictive values and likelihood ratios are most useful for assessing the value of reagent strip-detected hematuria and proteinuria in predicting the presence or absence of infection within subgroups of the populations ${ }^{(20)}$. So in this study will calculate predictive values, likelihood ratios, diagnostic efficiency, and area under the curve for more practical information. Quality control of reagent strip tests is fulfilled 
through control reference solutions which are available from manufacturers for some strips. The performance of most strip tests can also be controlled by checking regularly the strip reactions against those obtained by standard chemical tests. Control urines of known negative and positive reactions should also be prepared and tested with patient's specimens. Up-to-date manufacturers` literature must always be consulted regarding the quality control of different strips and the substances which may interfere with the various reactions ${ }^{(19)}$. This study used both chemical reagent strip and microscopic examination of urine samples to detect of S.haematobium eggs for 1000 patient samples to evaluate the validity of haematuria and proteinuria compared with a microscopic examination for the diagnosis of S.haematobium infection.

\section{Research objective:}

To evaluate the validity of haematuria and proteinuria by the chemical reagent strip compared with a microscopic examination for the diagnosis of S.haematobium infection.

\section{Material and methods}

\subsection{Study population and ethical consideration}

This study included 1000 patients attending the Ministry of health laboratory centers, in ElFayoum Governorate, these study subjects were randomly selected irrespective of the age-group and both genders were included. All the studied population was informed about the purpose of sample collection and their consent was obtained. Patients were free to refuse sample collection.

\subsection{Study design}

This research is a cross-sectional study designed to evaluate haematuria and proteinuria by the chemical reagent strip to determine urinary schistosomiasis in the study population. This study was targeting customers who came for laboratories of health centers for urine analysis.

\subsection{Collection and processing of urine samples}

Clean specimen bottles were labeled with the needed information and issued to the participating individuals whose informed consent was sought earlier, each patient was given a wide mouth screwcapped container into which to void urine. This was carried out between 10.00 am and $2.00 \mathrm{pm}$ when the ova count of S.haematobium is expected to be at its peak ${ }^{(21)}$. In this study, urinary schistosomiasis was defined as the presence of ova of S.haematobium in the urine.

\subsection{Urine microscopy}

Urine examination was carried out in the laboratory of health centers and by the researcher. Urine samples were examined for the presence of S.haematobium eggs as in the sedimentation method of Cheesbrough (2006) ${ }^{(22)}$. Each urine sample was mixed thoroughly with a glass rod and two samples were taken for each $10 \mathrm{ml}$ urine, one sample for sedimentation centrifugation and the other $10 \mathrm{ml}$ urine sample for the Nuclepore membrane filtration technique. The first $10 \mathrm{ml}$ transferred into a centrifuge tube and centrifuged at $2000 \mathrm{rpm}$ for 5 minutes at room temperature. The supernatant was then discarded and sediment was transferred to a microscope glass slide and covered with a coverslip. A drop of Lugol's Iodine was added onto the coverslip before the examination. Examination of the entire sediment was carried out using the $\mathrm{x} 10$ objective of a compound light microscope.

The second $10 \mathrm{ml}$ urine sample was examined using the Nuclepore membrane filtration technique for S.haematobium eggs detection as in the method of Cheesbrough (2009) ${ }^{(23)}$.

\subsection{Chemical reagent strip:}

For 1000 urine samples, chemical reagent strips were tested for the presence of protein and blood,(Teco diagnostics Anaheim, CA, the U.S.A. Which were the same strips used by health center laboratories).

-Blood: the test is based on the pseudoperoxidase action of hemoglobin and erythrocytes which 
catalysis the reaction of 3,3',5, 5'tetramethylbenzidine and buffered organic peroxide. The resulting colors range from orange to yellowgreen and dark green. Very high blood concentration may cause color development to continue to dark blue.

-Protein: this test is based on the protein error-ofindicator principle. At a constant $\mathrm{PH}$, the development of any green color is due to the presence of protein, colors range from yellow for a negative reaction to yellow-green and green to bluegreen for a positive reaction.

\section{Test procedure:}

a- Remove from the bottle only enough strips for immediate use and replace the cap tightly.

b- Completely immerse reagent areas of the strip in fresh, well-mixed urine. Remove the strip immediately to avoid dissolving out the reagent areas.

c- While removing, touch the side of the strip against the rim of the urine container to remove excess urine, blot the lengthwise edge of the strip on an absorbent paper towel to further remove excess urine and avoid running over (contamination from adjacent reagent pads).

d- Compare each reagent area to its corresponding color blocks on the color chart and read at the times specified, proper read time is critical for optimal results.

e- Obtain results by direct color chart comparison. Reagent areas may be read between 1-2 minutes for screening positive urine from negative urine, changes of color after 2 minutes are of no diagnostic value.

\subsection{Statistical analysis}

Results were collected, coded, tabulated, and analyzed through computer facilities using statistical methods S.haematobium infection was defined as any number of eggs greater than zero found in $10 \mathrm{ml}$ of urine, was performed to compare with haematuria and proteinuria by chemical reagent strip for Diagnosis of Schistosoma haematobium Infection. data analysis Correlation of the reagent strip results with the gold-standard parasitological data was done using diagnostic accuracy tests. Sensitivity, specificity, positive and negative predictive values (PPV and NPV), likelihood ratios for positive results (LR+) and diagnostic efficiency, and the area under the curve (AUC) were calculated. In this study, we considered using the sum of Nuclepore membranes filtration technique and Centrifugation sedimentation technique results as a gold standard to compare them with haematuria and proteinuria by chemical reagent strip ${ }^{(23)}$.

\section{Results}

Table (1): shows a diagnostic performance of haematuria, proteinuria by chemical reagent strips, and both as diagnostic methods for Schistosoma haematobium compared to microscopic examination techniques as the gold standard. In this study, we considered using the sum of Nuclepore membranes filtration technique and Centrifugation sedimentation technique results as a gold standard $^{(23)}$.

The results showed that haematuria by chemical reagent strips had a sensitivity of $27.8 \%$ and specificity of $63.4 \%$. When proteinuria was considered, sensitivity increased to $67.1 \%$ also specificity increased to $80.7 \%$. When both haematuria and proteinuria were considered, sensitivity was $45.2 \%$ and specificity $85.9 \%$. sensitivity in between the previous results. PPVs ranged between $6.13 \%$ and $22.9 \%$ whereas NPVs ranged between $91.1 \%$ and $96.6 \%$. As for diagnostic efficiency, it was best for both haematuria and proteinuria $(83.1 \%)$ followed by proteinuria $79.6 \%$ and least for haematuria $(60.7 \%)$, where the area under the curve (AUC) was sufficient for both haematuria and proteinuria 0.61 and good for proteinuria (0.71), where it was not useful for haematuria (0.48). 
Table (1): Diagnosis of S.haematobium infection using a chemical reagent strip compared to microscopic examination technique.

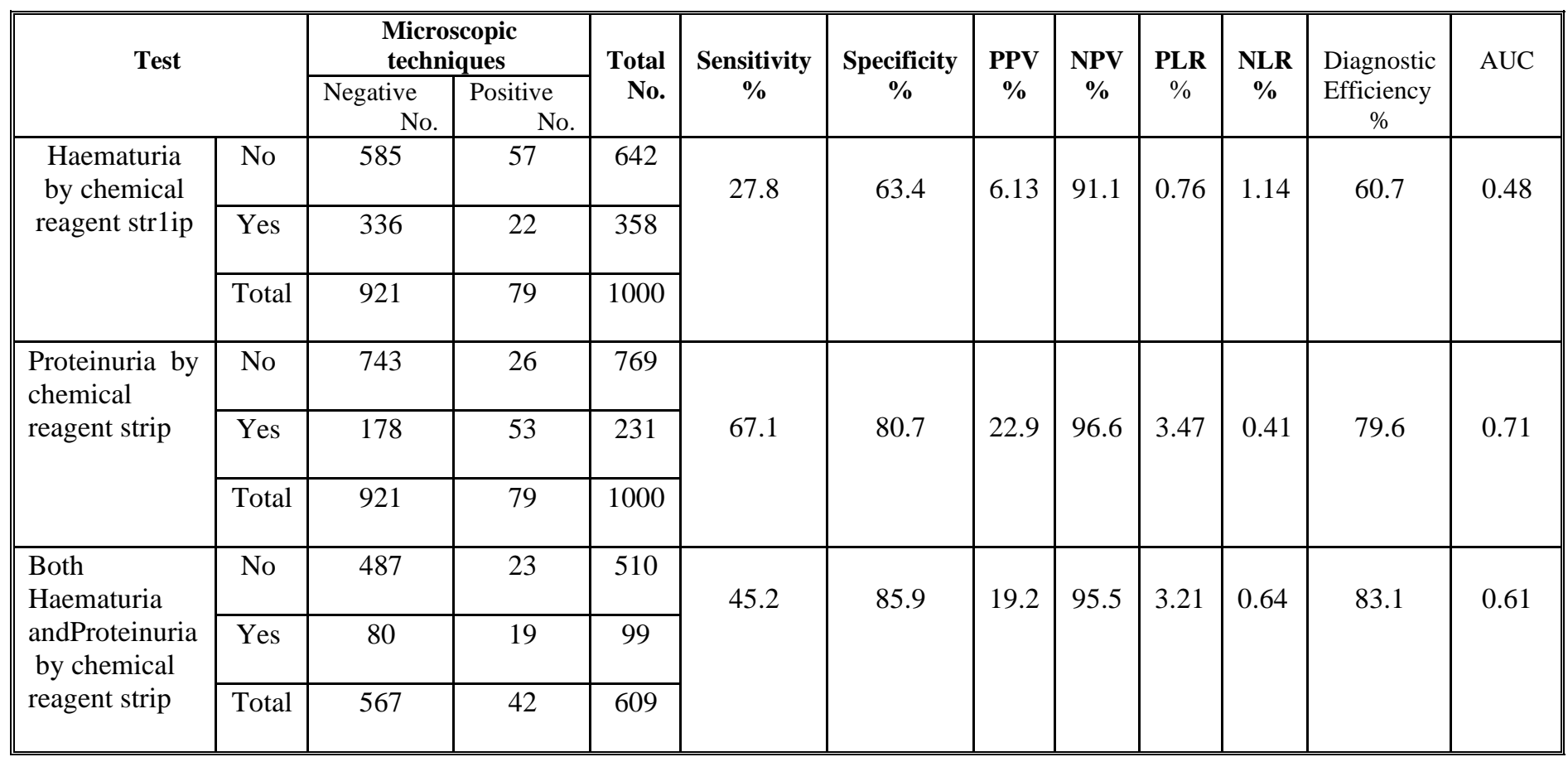

PPV, Positive predictive value; NPP, Negative predictive value; PLR, Likelihood ratio for positive results; NPL, Likelihood ratio for negative results; AUC Area under the curve.

\section{Discussion}

Reagent strips for microhaematuria and simple questionnaires for red urine are easy, cheap, and effective tools for the screening and rapid epidemiological assessment of urinary schistosomiasis ${ }^{(25)}$, such indirect diagnostic methods are less satisfactory for schistosomiasis intestinal or hepatic disease ${ }^{(26)}$. Since the early 1980s, reagent strip testing for microhaematuria has been used as an indirect diagnostic assay for Schistosoma haematobium ${ }^{(9)}$.

This study used the same chemical reagent strip which used in health center laboratories (Teco diagnostic USA), to detect blood and protein in the urine, most or nearly all previous studies showed blood in urine or microhaematuria is more important than protein and used chemical reagent strips to detect microhaematuria and not protein. But this study showed detect microhaematuria by chemical reagent strip has a sensitivity of $27.8 \%$, a specificity of $63.4 \%$, a positive predictive value of $6.13 \%$, and a negative predictive value of $91.1 \%$, while the protein in urine by chemical reagent strip had a sensitivity of $67.1 \%$, a specificity of $80.7 \%$, the positive predictive value of $22.9 \%$ and negative predictive value of $96.6 \%, \%$ ), where the area under the curve (AUC) was sufficient for both haematuria and proteinuria 0.61 and good for proteinuria( 0.71), where it was not useful for haematuria (0.48). By this study, the detection of proteinuria by chemical reagent strip has more sensitivity and more specificity than haematuria for diagnosis of S.haematobium infection. 
Poygensee $\mathrm{G}$ et al (2000), reported the validity of urine reagent strip reading which haematuria was showed a sensitivity of $64.9 \%$, specificity of $67.7 \%$, PPV of $54.7 \%$, and NPV of $57.5 \%$, but proteinuria showed a sensitivity of $12.2 \%$, a specificity of $91.3 \%$, PPV of $37.9 \%$ and NPV of $75.3 \%$ (27).

Nagi MA, (2005), in Yamen, evaluate the reagent strip, he showed that it was has a sensitivity of $56.5 \%$, a specificity of $57.3 \%$, PPV of $72.2 \%$, and NPV of $39.9 \%$ (28).

Michael D et al,(2007), used microhaematuria with reagent strips as a rapid assessment for urinary Schistosomiasis it showed a sensitivity of $85 \%$, a specificity of $98.5 \%$, PPV of $83.5 \%$, and NPV of $98.5 \%{ }^{(29)}$. The sensitivity of these strips for detection of microhaematuria is higher than that of urine filtration (Michael D. French, et al., 2007; Robinson et al., 2009) ${ }^{(29,30)}$. Some authors have argued that most of this background

microhaematuria is due to undetected S.haematobium explained by the lack of sensitivity of widely used diagnostic tools ${ }^{(31,32)}$.

In the 2015 Cochrane systematic review, it has been summarised that reagent strip testing for $S$. haematobium diagnosis has an overall sensitivity and specificity of $75 \%$ and $87 \%$, respectively ${ }^{(33)}$. where microhaematuria could not be associated with $S$. haematobium through reference microscopy $(33,34)$.

A study in Nigeria (2017) recorded that: Proteinuria and haematuria were observed to occur in $67.0 \%$ and $79.0 \%$ respectively. Males had higher infections and higher occurrences of proteinuria and haematuria $(72.5 \%, 88.2 \%$ respectively). There was no significant difference concerning gender $(\mathrm{P}<0.05)^{(35)}$

\section{Conclusion and Recommendations}

Most previous studies showed blood in urine or microhaematuria is more important than protein and used chemical reagent strips to detect microhaematuria and not protein. While this study showed detect of protein by chemical reagent strip is more important than microhaematuria by chemical reagent strip, and statistical parameters tested were higher with proteinuria than haematuria. The sensitivity, specificity, positive and negative predictive value, the likelihood ratio for positive results, diagnostic efficiency, and area under the curve all were higher in proteinuria than haematuria. The sum of proteinuria and haematuria had more specificity.

These strips suitable for initial mapping and screening of urinary schistosomiasis in the field. However, microhaematuria and proteinuria are nonspecific signs that could also result from other diseases such as urogenital infection, malignancy, immune system disorders, metabolic disorders, and trauma. In an infield study, screening in a high endemic area with schistosomiasis using strips for detection of both proteinuria and haematuria is more valid than the detection of haematuria alone.

In this study, we demonstrate that the use of urine reagent strips for both proteinuria and haematuria might be considered for the diagnosis of $S$. haematobium where microscopy is unavailable.

\section{Conflict of interest}

There are no conflicts of interest.

\section{Financial support and sponsorship}

This research did not receive any specific grant from funding agencies in the public, commercial, or not-for-profit sectors.

\section{References:}

1. Houmsou, R.S., Amuta, E.U., and Sar, T.T. (2012). Profile of an epidemiological study of urinary schistosomiasis in two local government areas of Benue State, Nigeria. International Journal of Medical Biomedical Research, 1(1):39-48

2. Abdel-Wahab M.F., Esmat G., Medhat E., Narooz S., Ramzy I., El-Boraey Y., Strickland G.T. 2000. The epidemiology of 
schistosomiasis in Egypt: Menofia Governorate. Am. J. Trop. Med. Hyg. 62(2 suppl):28 - 34 .

3. El-Khoby T., Galal N., Fenwick A., Barakat R., El- Hawey A., Nooman Z., Habib M., AbdelWahab

F., Gabr N.S., Hammam H.M. 2000. The epidemiology of schistosomiasis in Egypt: summary findings in nine governorates. Am. J. Trop. Med. Hyg. 62(2 suppl):88-99.

4. Ahmed A. Yameny. The evolving schistosomiasis agenda 2017- 2020 in Egypt: Moving from control toward final elimination. J Biosci App Res .2017;3(2): 48- 54.

5. WHO. 2007. Report of intercountry meeting on a strategy to eliminate schistosomiasis from the Eastern Mediterranean Region. Muscat, Oman.6-8 November.

6. Ahmed A. Yameny. Schistosomiasis haematobium prevalence and risk factors in ElFayoum Governorate, Egypt. J Biosci App Res. 2017;3(3):191-201.

7. Colley D., Binder S., Campbell C. 2013. A five-country evaluation of a point-of-care circulating cathodic antigen urine assay for the prevalence of Schistosoma mansoni. Am. J. Trop. Med. Hyg. 88:426-432.

8. Davidson RN, Houston S, Kiire CF. Schistosomal periportal fibrosis in Zimbabwe: use of ultrasound in patients with oesophageal varices. Trans R Soc Trop Med Hyg 1991; 85: 380-382.

9. Mott KE, Dixon H, Osei-Tutu E, England EC, Ekuek, Tekle A. Evaluation of reagent strips in urine tests for detection of Schistosoma haematobium: a comparative study in Ghana and Zambia. Bull World Health Organ 1985; 63: 125-33.

10. Ndhlovu P, Cadman H, Gundersen $\mathrm{S}$, Vennervuld BJ, Friis H, Christensen NO, et al. Circulation anodic antigen (CAA) levels in different age groups in Zimbabwean rural community endemic for schistosoma haematobium determined using the magnetic beads antigen-capture enzyme-linked immunoassay. Am J Trop Hyg 1996; 54(5): 537-542.

11. Savioli L, Mott KE. Urinary schistosomiasis on Pemba Island: Low-cost diagnosis for control in a primary healthcare setting. Parasitol Today 1989; 5: 333-337.

12. Savioli L, Hatz C, Dixon H, Kisumkuu UM, Mott KE. Control of morbidity due to $\mathrm{S}$. haematobium on Pemba Island: egg excretion and haematuria as indicators of infection. Am J Trop Med Hyg 1990; 43: 289-295.

13. Feldmeier H. Diagnosis. In: Human schistosomiasis. Walling Ford, England, CAB international, 1993.

14. Doehring E, Feldmeire H, Doffalla AA, Ehrich JHH, Vester U, Proggensee U. Intermittent chemotherapy with trichlorfon (metrifonate) reverse proteinuria, hematuria, and leukocyturia in urinary schistosomiasis: results of a threeyear field study. J Infect Dis 1984; 149: 615620.

15. Etten LV, Kremsner PG, Karigger FW, Deelder AM. Day-to-day variation of egg output and schistosome circulating antigens in urine of schistosoma haematobium infected school children from Gabon and follow-up after chemotherapy. Am J Trop Med Hyg 1997; 57(3): 337-341.

16. Murare HM, Taylar P, Haematuria and proteinuria during schistosomiasis heamatobium infection; relationship to intensity of infection and the value of chemical reagent strips for pre-and post-treatment diagnosis. Trans R Soc Trop Med Hyg 1987; 81: 426-430.

17. Feldmeier H. A synoptic inventory of needs for research on women and tropical parasitic diseases. II. Gender-related biases in the diagnosis and morbidity assessment of schistosomiasis in women. Acta Tropica 1993; 55: 139-169. 
18. Cheesbrough M. District laboratory practice in tropical countries. Cambridge: 1999 (part 2); 105-115.

19. Cheesebrough M. district laboratory practice in tropical countries. Cambridge; 1999 (part 1); 236-239, 372-374.

20. Hammad TA, Gaber NS, Talaat MM, Orieby A, Shawky E, Strickland T, Hematuria and proteinuria as predictors of schistosoma haematobium infection. Am J Trop Med Hyg 1997; 57(3): 363-367.

21. Eyong ME, Ikepeme EE, Ekanem EE. Relationship between Schistosoma haematobium infection and urinary tract infection among children in South Eastern, Nigeria. Niger Postgrad Med J2008;15:89-93.

22. Cheesbrough M. District laboratory practice in tropical countries part 2. United Kingdom: Cambridge University Press. Cambridge. 2006; 323-41.

23. Cheesbrough M. Laboratory-practice-intropical-countries-part-1. United Kingdom: Cambridge University Press. Cambridge. 2009;p.357

24. Ahmed A. Yameny. Evaluation of Nuclepore Filteration Technique for Diagnosis of Schistosoma haematobium Infection. J Biosci App Res. 2015;1(6):335-341.

25. Lengeler C, Utzinger J, Tanner M. Screening for schistosomiasis with questionnaires. Trends Parasitol 2002; 18: 375-77.

26. Tan HZ, Yang MX, Wu ZG, et al. Rapid screening method for Schistosoma japonicum infection using questionnaires in flood area of the People's Republic of China. Acta Tropica 2004; 90: 1-9.

27. G. Poggensee, L. Krantz, I. Kiwelu, H. Feldmeier. Screening of Tanzanian women of childbearing age for urinary schistosomiasis: validity of urine reagent strip readings and selfreported symptoms. Bulletin of the World Health Organization, 2000, 78(4).
28. Nagi MAM. Evaluation of a programme for control of Schistosoma haematobium infection in Yemen. Eastern Mediterranean Health Journal volume 11, 2005.

29. Michael D. French, David Rollinson, MariaGloria Basanez, Ali F. Mgeni, I. Simba Khamis and J. Russel Stothard. School-based control of urinary schistosomiasis on Zanzibar, Tanzania: Monitoring microhaematuria with reagent strips as a rapid urological assessment. J. Ped. Urol. 2007; 3(5):364-368.

30. Robinson E., Picon D., Sturrock H. J., Sabasio A., Lado M., Kolaczinski J., Brooker S. 2009. The performance of haematuria reagent strips for the rapid mapping of urinary schistosomiasis: field experience from Southern Sudan. Trop. Med. Int. Health 14(12):14841487. DOI: $10.1111 /$ j.1365-3156.2009.02407

31. Bergquist R, Johansen MV, Utzinger J. Diagnostic dilemmas in helminthology: what tools to use and when? Trends Parasitol. 2009;25:151-6.

32. Utzinger J, Becker SL, van Lieshout L, van Dam GJ, Knopp S. New diagnostic tools in schistosomiasis. Clin Microbiol Infect. 2015;21:529-42.

33. Ochodo EA, Gopalakrishna G, Spek B, Reitsma JB, van Lieshout L, Polman K, et al. Circulating antigen tests and urine reagent strips for diagnosis of active schistosomiasis in endemic areas. Cochrane Database Syst Rev. 2015;3:CD009579.

34. King $\mathrm{CH}$, Bertsch D. Meta-analysis of urine heme dipstick diagnosis of Schistosoma haematobium infection, including lowprevalence and previously-treated populations. PLoS Negl Trop Dis. 2013;7:e2431.

35. Juliana Eze Elom1, Oliver O. Odikamnoro1, Agwu Ulu Nnachi, Ifeanyi Ikeh1, John O. Nkwuda. Variability of Urine Parameters in Children Infected with Schistosoma haematobium Ukawu Community, Onicha Local Government Area, Ebonyi State, Nigeria. Afr., J. Infect. Dis. (2017) 11 (2): 10-16. 